\title{
On Carleman Formulas for the Dolbeault Cohomology
}

\author{
Mauro Nacinovich \\ Bert-Wolfgang Schulze \\ Dipartimento di Matematica \\ Institut für Mathematik \\ Via F. Buanaroti 2 \\ Universität Potsdam \\ 56127 Pisa \\ Postfach 601553 \\ Italy \\ 14415 Potsdam \\ Germany
}

\author{
Nikolai N. Tarkhanov* \\ Institute of Physics \\ Russian Academy of Sciences \\ Akademgorodok \\ 660036 Krasnoyarsk \\ Russia
}

February 21, 1998

\footnotetext{
${ }^{*}$ Supported by the Max-Planck Gesellschaft.
} 


\begin{abstract}
We discuss the Cauchy problem for the Dolbeault cohomology in a domain of $\mathbb{C}^{n}$ with data on a part of the boundary. In this setting we introduce the concept of a Carleman function which proves useful in the study of uniqueness. Apart from an abstract framework we show explicit Carleman formulas for the Dolbeault cohomology.

AMS subject classification: primary: 32F 20; secondary: 58G05. las.

Key words and phrases: $\bar{\partial}$-operator, cohomology, integral formu-
\end{abstract}




\section{Contents}

Introduction $\quad 5$

1 Koppelman kernel $\quad 6$

2 Abstract framework $\quad 8$

3 Carleman formula $\quad 10$

4 Support function $\quad 12$

$\begin{array}{ll}\text { References } & 13\end{array}$ 
Nacinovich, Schulze, Tarkhanov 


\section{Introduction}

Analytic continuation is a central problems in complex analysis and its applications. Assume we are given a class of analytic functions in a domain $\mathcal{D}$ of $\mathbb{C}^{n}$ which are well behaved on the boundary of $\mathcal{D}$. A subset $S$ of $\partial \mathcal{D}$ is said to be a set of uniqueness for this class if any function of the class vanishing on $S$ is zero in the whole domain $\mathcal{D}$. It is well-known that in general analytic functions on $\mathcal{D}$ do not depend continuously on their values on a uniqueness set unless $S$ contains the Shilov boundary of $\mathcal{D}$. For this reason it is impossible in general to recover the values of an analytic function of the class from its values on $S$ by a "nice" integral formula. The instability should result in integral representation formulas involving either a passage to the limit or a non-compact domain of integration. However, the functional evaluating the holomorphic functions at the points of $\mathcal{D}$ may still be continuous with respect to some two-norm convergence which requires, along with convergence on $S$, boundedness in all of $\mathcal{D}$. Such formulas are referred to as 'Carleman formulas.' For the existence of a Carleman formula it is not sufficient that $S$ be a set of uniqueness for the corresponding class. In fact, such a formula exists if and only if $S$ is a set of conditional stability (cf. [AT90]).

The Carleman formulas for holomorphic functions of one and several complex variables bear many applications both in the complex analysis itself and in the natural science. As but one example of these we recall the problem of supersolvability of physical devices. For a systematic treatment containing most of what is known on Carleman formulas and their applications we refer the reader to Aizenberg [Aiz93].

In higher-dimensional complex analysis $\bar{\partial}$-closed differential forms and especially their Dolbeault cohomology classes play a very important role, aside from holomorphic functions. A cohomology class is a good substitute of a holomorphic function; local triviality near a point of the boundary substitutes analytic continuation, although a lack of localisability makes the notion of extendibility of cohomology classes more complicate.

Thus, the Cauchy problem for the Dolbeault cohomology in a domain with data on a part of the boundary generalises that of analytic continuation. It was treated by Andreotti and Hill [AH72a] who reduced the questions of uniqueness and existence to the vanishing of some cohomology of the Dolbeault complex in the domain. The problem arises of finding a suitable analog of Carleman formulas for holomorphic functions for the Dolbeault cohomology classes. To this end, we first need an explicit description of subsets $S$ of the boundary with the property that each $\bar{\partial}$-closed differential form $u$ in $\mathcal{D}$ which is $\bar{\partial}$-exact near $S$ is actually $\bar{\partial}$-exact on all of $\mathcal{D}$. This question is at present far from being solved. We will restrict our 
consideration to open subsets of the boundary.

A heuristic explanation of the procedure involved might be as follows. Suppose that the boundary of $\mathcal{D}$ is "good" in some sense away from the set $S$. By a "good" boundary we mean a hypersurface which is either strictly pseudoconvex or on which the Levi form has a prescribed number of positive eigenvalues. Then, were we able to solve the equation $\bar{\partial} u=f$ near $S$ in $\mathcal{D}$, the "good" structure of $\partial \mathcal{D} \backslash S$ allow us to find a solution it in the whole domain. However, things seem to go in a slightly different way and we have to impose on $\partial \mathcal{D} \backslash S$ a pseudoconcavity condition rather than one of pseudoconvexity.

Moreover, if $S$ is a set of uniqueness for some group of Dolbeault cohomology in the domain $\mathcal{D}$, we are interested in finding integral formulas which allow us to represent any $\bar{\partial}$-closed differential form $u$ in $\mathcal{D}$, up to a $\bar{\partial}$-exact form, via the cohomology class defined by $u$ on $S$. For this purpose, we use Koppelman's extension of the Martinelli-Bochner formula to differential forms (cf. [Kop67]). The strategy is to represent our $\bar{\partial}$-closed differential form in $\mathcal{D}$ as the sum of two integrals, the first of the two being over the boundary and the second one $\bar{\partial}$-exact in $\mathcal{D}$. Next we split the boundary integral as the sum of one over $S$ and one over its complement in $\partial \mathcal{D}$. In this process we approximate the Koppelman kernel on $\partial \mathcal{D} \backslash S$ by $\bar{\partial}$-closed differential forms in a neighborhood of the closure of the domain. In this way we obtain a representation that we refer to as a generalised Carleman formula for the Dolbeault cohomology.

\section{Koppelman kernel}

For $n$-dimensional vectors $v_{1}, \ldots, v_{N}$ with entries in a ring and non-negative integers $n_{1}, \ldots, n_{N}$ with $n_{1}+\ldots+n_{N}=n$, we denote by $D_{n_{1}, \ldots, n_{N}}\left(v_{1}, \ldots, v_{N}\right)$ the determinant of order $n$ whose first $n_{1}$ columns are $v_{1}$, the next $n_{2}$ columns are $v_{2}$ etc., the last $n_{N}$ columns are $v_{N}$. We compute the determinant by columns, i.e., we $\operatorname{define} \operatorname{det}\left(v_{i j}\right)=\sum_{I}(-1)^{\varepsilon_{I}} v_{i_{1} 1} \ldots v_{i_{n} n}$ where $\varepsilon_{I}$ denotes the signature of the permutation $I=\left(i_{1}, \ldots, i_{n}\right)$ of the integers $(1, \ldots, n)$.

Let $v=v(z, \zeta, t)$ be a smooth function on $O \times[0,1]$ with values in $\mathbb{C}^{n}$, $O$ being an open set not intersecting the diagonal $\{z=\zeta\}$ in $\mathbb{C}_{z}^{n} \times \mathbb{C}_{\zeta}^{n}$. Fix $0 \leq p \leq n$. Consider the double differential forms $K_{q}^{(p)}(w)$ of bidegree $(p, q-1)$ in $z$ and $(n-p, n-q)$ in $\zeta, t$ given by

$$
\begin{aligned}
& K_{q}^{(p)}(v)=\frac{(-1)^{q+(n-p)(q-1)}}{(2 \pi i)^{n} n !}\left(\begin{array}{c}
n \\
p
\end{array}\right)\left(\begin{array}{c}
n-1 \\
q-1
\end{array}\right) \\
& \quad \times D_{p, n-p}(\partial z, \partial \zeta) \wedge D_{1, q-1, n-q}\left(v, \bar{\partial}_{z} v,\left(\bar{\partial}_{\zeta}+d_{t}\right) v\right),
\end{aligned}
$$

for $1 \leq q \leq n$, and $K_{0}^{(p)} \equiv K_{n+1}^{(p)} \equiv 0$.

The double forms (1.1) were first introduced by Koppelman [Kop67]. Here we rehearse some elementary properties of these forms. 
Lemma 1.1 For each smooth function $f$ on $O \times[0,1]$, we have the equality $K_{q}^{(p)}(f v)=f^{n} K_{q}^{(p)}(v)$.

Proof. Indeed, if $\partial$ is one of the differentials $\bar{\partial}_{z}, \bar{\partial}_{\zeta}$ and $d_{t}$, then we get $\partial(f v)=(\partial f) v+f \partial v$. As the vector $(\partial f) v$ is proportional to $v$, it gives no contribution to the last determinant on the right-hand side of (1.1). This proves the lemma.

In particular, if $v$ satisfies $\langle v, \zeta-z\rangle \neq 0$ pointwise on the set $O \times[0,1]$, then

$$
K_{q}^{(p)}\left(\frac{v}{\langle v, \zeta-z\rangle}\right)=\frac{1}{\langle v, \zeta-z\rangle^{n}} K_{q}^{(p)}(v)
$$

where $\langle\cdot, \cdot\rangle$ denotes the standard bilinear form $\mathbb{C}^{n} \otimes \mathbb{C}^{n} \rightarrow \mathbb{C}$. Thus, when considering a vector-valued function $v$ with the property that $\langle v, \zeta-z\rangle \neq 0$ on $O \times[0,1]$, after multiplication by a non-zero function we may actually assume that $\langle v, \zeta-z\rangle=1$.

Lemma 1.2 Suppose $v$ satisfies $\langle v, \zeta-z\rangle=1$ on $O \times[0,1]$. Then, we have

$$
\left(\bar{\partial}_{\zeta}+d_{t}\right) K_{q+1}^{(p)}(v)=(-1)^{p+q} \bar{\partial}_{z} K_{q}^{(p)}(v)
$$

Proof. Cf. for instance Lemma 1.2 in Aizenberg and Dautov [AD75] and elsewhere.

Note that if $v_{j}=v_{j}(z, \zeta), j=1,2$, are smooth functions on $O$ with values in $\mathbb{C}^{n}$, both satisfying $\left\langle v_{j}, \zeta-z\right\rangle=1$ on $O$, then the linear homotopy $v_{t}=(1-t) v_{0}+t v_{1}$ between them still satisfies $\left\langle v_{t}, \zeta-z\right\rangle=1$ on the set $O \times[0,1]$.

Lemma 1.3 Let $v$ satisfy $\langle v, \zeta-z\rangle=1$ on $O \times[0,1]$. Write $v_{0}$ and $v_{1}$ for the values of $v$ at $t=0$ and $t=1$, respectively. Then

$$
K_{q+1}^{(p)}\left(v_{1}\right)-K_{q+1}^{(p)}\left(v_{0}\right)=\bar{\partial}_{z} I_{q+1}^{(p)}(v)-(-1)^{p+q} \bar{\partial}_{\zeta} I_{q+2}^{(p)}(v)
$$

on the set $O$, where $\left.I_{i}^{(p)}(v)=(-1)^{p+q} \int_{0}^{1} d t\right\rfloor K_{i-1}^{(p)}(v) d t$.

Proof. It suffices to integrate equality (1.2) over $t \in[0,1]$ and take into account that

$$
\left.\left.\bar{\partial}_{\zeta} \int_{0}^{1} d t\right\rfloor K_{q+1}^{(p)}(v) d t=-\int_{0}^{1} d t\right\rfloor \bar{\partial}_{\zeta} K_{q+1}^{(p)}(v) d t
$$

because $\bar{\partial}_{\zeta}$ and $d_{t}$ anticommute.

There is a universal solution to the equation $\langle v, \zeta-z\rangle=1$ outside of the diagonal in $\mathbb{C}_{z}^{n} \times \mathbb{C}_{\zeta}^{n}$, given by $v_{1}(z, \zeta)=(\bar{\zeta}-\bar{z}) /|\zeta-z|^{2}$ for $z \neq \zeta$. Under this choice of $v$, the double forms $K_{q}^{(p)}(v)$ fit together to give a fundamental solution of convolution type to the Dolbeault complex on $\mathbb{C}^{n}$. 
Lemma 1.4 Let $\mathcal{D}$ be a bounded domain in $\mathbb{C}^{n}$ with a piecewise smooth boundary and $u \in C^{1}\left(\Lambda^{p, q} T_{\mathbb{C}}^{*} \overline{\mathcal{D}}\right)$. Then,

$$
-\int_{\partial \mathcal{D}} u \wedge K_{q+1}^{(p)}\left(v_{1}\right)+\int_{\mathcal{D}} \bar{\partial} u \wedge K_{q+1}^{(p)}\left(v_{1}\right)+\bar{\partial} \int_{\mathcal{D}} u \wedge K_{q}^{(p)}\left(v_{1}\right)=\chi_{\mathcal{D}} u,
$$

where $\chi_{\mathcal{D}}$ is the characteristic function of $\mathcal{D}$.

Proof. Cf. the original paper of Koppelman [Kop67]. For a thorough treatment we also refer the reader to [AD75].

\section{Abstract framework}

In the sequel, we denote by $\mathcal{D}$ a bounded domain in $\mathbb{C}^{n}$ with a piecewise smooth boundary.

Let $S$ be an open subset of $\partial \mathcal{D}$ with a piecewise smooth boundary. Assume moreover that $\partial \mathcal{D} \backslash S$ is smooth. Then we can find an open neighbourhood $U$ of $\partial \mathcal{D} \backslash S$ and a smooth real-valued $\varrho$ in $U$ such that $\nabla \varrho \neq 0$ on $U \cap \partial \mathcal{D}$ and $\mathcal{D} \cap U=\{z \in U: \varrho(z)<0\}$. We call such a function $\varrho$ a local defining function of $\mathcal{D}$ near $\partial \mathcal{D} \backslash S$.

Fix $0 \leq q \leq n-1$. Recall that the part $\partial \mathcal{D} \backslash S$ of $\partial \mathcal{D}$ is called strictly $q$-pseudoconcave if the Levi form of $\varrho$ has at least $q$ negative eigenvalues at each point of $\partial \mathcal{D} \backslash S$. This property is independent of the choice of the local defining function. Note that 0 -pseudoconcavity imposes no condition on the Levi form.

We may also regard $\partial \mathcal{D} \backslash S$ as a part of the boundary of the complement of $\overline{\mathcal{D}}$. Then, $-\varrho$ is a local defining function for $\mathbb{C}^{n} \backslash \overline{\mathcal{D}}$ close to $\partial \mathcal{D} \backslash S$. A $q$-pseudoconcavity assumption for $\varrho$ implies that the Levi form of $-\varrho$ has at least $q$ positive eigenvalues at each point of $\partial \mathcal{D} \backslash S$. Hence, at most $n-1-q$ eigenvalues may be negative or zero. In this case we say that $\partial \mathcal{D} \backslash S$ is strictly $(n-1-q)$-pseudoconvex. The usual 'strict pseudoconvexity' thus corresponds to the 'strict 0 -pseudoconvexity'.

The following result is a kind of Lemma 4.5.3 of [Tar95] in the case of the Dolbeault complex.

Lemma 2.1 Let $0 \leq q \leq n-1$. Suppose $\partial \mathcal{D} \backslash S$ is strictly $q$-pseudoconcave. Then, there are a neighbourhood $U$ of $\partial \mathcal{D} \backslash S$ in $\mathbb{C}^{n}$ and smooth double differential forms $R_{q+1}^{(p)}(z, \zeta)$ and $P_{q+1}^{(p)}(z, \zeta)$ on $(U \cap \mathcal{D}) \times(U \backslash \mathcal{D})$, such that

$$
\begin{aligned}
K_{q+1}^{(p)}\left(v_{1}\right) & =R_{q+1}^{(p)}(z, \zeta)+(-1)^{p+q} \bar{\partial}_{z} P_{q+1}^{(p)}(z, \zeta), \\
\bar{\partial}_{\zeta} R_{q+1}^{(p)}(z, \zeta) & =0 .
\end{aligned}
$$

Note that $R_{q+1}^{(p)}(z, \zeta)$ and $P_{q+1}^{(p)}(z, \zeta)$ should be of bidegree $(p, q)$ and $(p, q-1)$ in $z$, respectively, and $(n-p, n-q-1)$ in $\zeta$.

Proof. Without loss of generality we may assume that the set $\partial \mathcal{D} \backslash S$ is connected. 
Since the Levi form of $\varrho$ has at least $q$ negative eigenvalues at each point of the compact set $\partial \mathcal{D} \backslash S$, this is still true in some neighbourhood of $\partial \mathcal{D} \backslash S$ on the boundary. It follows that there is a neighbourhood $U^{\prime}$ of $\partial \mathcal{D} \backslash S$ in $\mathbb{C}^{n}$ and a smooth function $\varrho^{\prime}$ in $U^{\prime}$ agreeing with - $\varrho$ near $\partial \mathcal{D} \backslash S$, such that $\Omega=\left\{z \in U^{\prime}: \varrho^{\prime}(z)<0\right\}$ is a domain with a compact closure in $U^{\prime},\left.\nabla \varrho^{\prime}\right|_{\partial \Omega} \neq 0$ and the Levi form of $\varrho^{\prime}$ has at least $q$ positive eigenvalues at each point of $\partial \Omega$.

Thus, $\Omega$ is a strictly $(n-1-q)$-pseudoconvex domain in $\mathbb{C}^{n}$ and the boundary of $\Omega$ intersects $\partial \mathcal{D}$ in a set containing a neighbourhood of $\partial \mathcal{D} \backslash S$. Set $U=(\mathcal{D} \backslash \bar{\Omega}) \cup(\bar{\Omega} \backslash \mathcal{D})$, then $\partial \mathcal{D} \backslash S$ lies in the interior of $U$ and

$$
\begin{aligned}
U \cap \mathcal{D} & =\mathcal{D} \backslash \bar{\Omega} \\
U \backslash \mathcal{D} & =\bar{\Omega} \backslash \mathcal{D} .
\end{aligned}
$$

By [AG62], the Dolbeault cohomology groups $H^{i}\left(\mathcal{E}\left(\Lambda^{p \cdot} \cdot T_{\mathbb{C}}^{*} \Omega\right)\right)$ are finitedimensional for $i \geq n-q$. Moreover, since $\Omega \subset \mathbb{C}^{n}$, they are actually zero (cf. [Lau75]). Note that this fact still holds if we replace $\Omega$ by the closure of $\Omega$.

For each fixed $z \in \mathcal{D} \backslash \bar{\Omega}$, the double differential form $K_{1}^{(p)}\left(v_{1}\right)$ is $\bar{\partial}$ closed in $\zeta \in \Omega$, as is clear from Lemma 1.2. Since $H^{n-1}\left(\mathcal{E}\left(\Lambda^{p \cdot \cdot} T_{\mathbb{C}}^{*} \Omega\right)\right)=0$, it follows that there is a double differential form $P_{2}^{(p)}(z, \zeta)$ of bidegree $(p, 0)$ in $z$ and $(n-p, n-2)$ in $\zeta$, such that $K_{1}^{(p)}\left(v_{1}\right)=\bar{\partial}_{\zeta} P_{2}^{(p)}(z, \zeta)$ for all $\zeta \in \Omega$. Combining a clever argument of Kerzman [Ker72] with a priori estimates for the solution of the $\bar{\partial}$-Neumann problem given in [AH72b], we can even ensure that $P_{2}^{(p)}(z, \zeta)$ be smooth for $(z, \zeta) \in(\mathcal{D} \backslash \Omega) \times \bar{\Omega}$ away from the diagonal $z=\zeta$.

Set $R_{2}^{(p)}(z, \zeta)=K_{2}^{(p)}\left(v_{1}\right)-(-1)^{p+1} \bar{\partial}_{z} P_{2}^{(p)}(z, \zeta)$. Using Lemma 1.2 we deduce that

$$
\begin{aligned}
\bar{\partial}_{\zeta} R_{2}^{(p)}(z, \zeta) & =\bar{\partial}_{\zeta} K_{2}^{(p)}\left(v_{1}\right)-(-1)^{p+1} \bar{\partial}_{z} \bar{\partial}_{\zeta} P_{2}^{(p)}(z, \zeta) \\
& =\bar{\partial}_{\zeta} K_{2}^{(p)}\left(v_{1}\right)-(-1)^{p+1} \bar{\partial}_{z} K_{1}^{(p)}\left(v_{1}\right) \\
& =0
\end{aligned}
$$

for all $(z, \zeta) \in(\mathcal{D} \backslash \bar{\Omega}) \times \Omega$. As $H^{n-2}\left(\mathcal{E}\left(\Lambda^{p, \cdot} T_{\mathbb{C}}^{*} \Omega\right)\right)=0$, we can apply the above argument again, with $K_{1}^{(p)}\left(v_{1}\right)$ replaced by $R_{2}^{(p)}(z, \zeta)$, to obtain a double differential form $P_{3}^{(p)}(z, \zeta)$ of bidegree $(p, 1)$ in $z$ and $(n-p, n-3)$ in $\zeta$, such that $R_{2}^{(p)}(z, \zeta)=\bar{\partial}_{\zeta} P_{3}^{(p)}(z, \zeta)$ for all $\zeta \in \Omega$. Once again we may find $P_{3}^{(p)}(z, \zeta)$ smooth outside of the boundary diagonal in $(\mathcal{D} \backslash \Omega) \times \bar{\Omega}$.

Continuing this process as far as the strict $(n-1-q)$-pseudoconvexity of $\Omega$ allows, we construct double differential forms $P_{i}^{(p)}(z, \zeta), i=2, \ldots, q+1$, on $(\mathcal{D} \backslash \bar{\Omega}) \times \bar{\Omega}$, such that

$$
K_{i}^{(p)}\left(v_{1}\right)=\bar{\partial}_{\zeta} P_{i+1}^{(p)}(z, \zeta)+(-1)^{p+i-1} \bar{\partial}_{z} P_{i}^{(p)}(z, \zeta)
$$




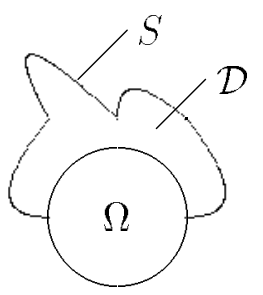

Fig. 1: A typical set of uniqueness for the Dolbeault cohomology.

for $i=1, \ldots, q$. Letting $R_{q+1}^{(p)}(z, \zeta)=K_{q+1}^{(p)}\left(v_{1}\right)-(-1)^{p+q} \bar{\partial}_{z} P_{q+1}^{(p)}(z, \zeta)$, we get, by Lemma 1.2,

$$
\begin{aligned}
\bar{\partial}_{\zeta} R_{q+1}^{(p)}(z, \zeta) & =\bar{\partial}_{\zeta} K_{q+1}^{(p)}\left(v_{1}\right)-(-1)^{p+q} \bar{\partial}_{z} \bar{\partial}_{\zeta} P_{q+1}^{(p)}(z, \zeta) \\
& =\bar{\partial}_{\zeta} K_{q+1}^{(p)}\left(v_{1}\right)-(-1)^{p+q} \bar{\partial}_{z} K_{q}^{(p)}\left(v_{1}\right) \\
& =0
\end{aligned}
$$

for all $(z, \zeta) \in(\mathcal{D} \backslash \bar{\Omega}) \times \Omega$. This completes the proof.

The proof actually shows that if $\mathcal{D}$ lies in the complement of a strictly $(n-q-1)$-pseudoconvex domain $\Omega$ and the boundary of $\mathcal{D}$ intersects $\partial \Omega$ in the complement of $S$, then the decomposition (2.1) is valid for all $z \in \mathcal{D}$ and $\zeta \in \bar{\Omega}$ (see Fig. 1).

\section{Carleman formula}

Using the double differential form $P_{q+1}^{(p)}(z, \zeta)$ constructed in Lemma 2.1, we may introduce a $\bar{\partial}$-homotopy operator

$$
h_{q}^{(p)} u(z)=-\int_{\partial \mathcal{D} \backslash S} u \wedge(-1)^{p+q} P_{q+1}^{(p)}(z, \cdot)+\int_{\mathcal{D}} u \wedge K_{q}^{(p)}\left(v_{1}\right), \quad z \in U \cap \mathcal{D},
$$

on differential forms $u$ of bidegree $(p, q)$ in $\mathcal{D}$ continuous up to the part $\partial \mathcal{D} \backslash S$ of the boundary. The interest of the operator $h_{q}^{(p)}$ lies in the fact that we obtain $\bar{\partial} h_{q}^{(p)} u=u$ in $U \cap \mathcal{D}$, provided $u$ is $\bar{\partial}$-closed and vanishes on $S$.

Theorem 3.1 Let $R_{q+1}^{(p)}(\epsilon ; z, \zeta), \epsilon>0$, be a family of double differential forms on $(U \cap \mathcal{D}) \times \overline{\mathcal{D}}, \bar{\partial}$-closed in $\zeta \in \mathcal{D}$ and approximating $R_{q+1}^{(p)}(z, \zeta)$ uniformly in $\zeta \in \partial \mathcal{D} \backslash S$ when $\epsilon \rightarrow 0$, for fixed $z \in U \cap \mathcal{D}$. If $u \in C^{1}\left(\Lambda^{p, q} T_{\mathbb{C}}^{*} \overline{\mathcal{D}}\right)$ is $\bar{\partial}$-closed, then

$$
u(z)=-\lim _{\epsilon \rightarrow 0} \int_{S} u \wedge\left(K_{q+1}^{(p)}\left(v_{1}\right)-R_{q+1}^{(p)}(\epsilon ; z, \cdot)\right)+\bar{\partial} h_{q}^{(p)} u(z), \quad z \in U \cap \mathcal{D} .
$$


Proof. Indeed, since $R_{q+1}^{(p)}(\epsilon ; z, \zeta)$ is $\bar{\partial}$-closed in $\zeta$ in the domain $\mathcal{D}$, for each $z \in U \cap \mathcal{D}$, we conclude from (1.4) and Stokes' formula that

$$
u(z)=-\int_{\partial \mathcal{D}} u \wedge\left(K_{q+1}^{(p)}\left(v_{1}\right)-R_{q+1}^{(p)}(\epsilon ; z, \cdot)\right)+\bar{\partial} \int_{\mathcal{D}} u \wedge K_{q}^{(p)}\left(v_{1}\right)
$$

if $z \in U \cap \mathcal{D}$. The boundary integral can be written as the sum of two integrals, one over $S$ and the other over $\partial \mathcal{D} \backslash S$. When integrating with respect to $\zeta \in \partial \mathcal{D} \backslash S$, we may invoke the decomposition of $K_{q+1}^{(p)}\left(v_{1}\right)$ given by (2.1). Hence it follows that

$$
\begin{aligned}
u(z)= & -\int_{S} u \wedge\left(K_{q+1}^{(p)}\left(v_{1}\right)-R_{q+1}^{(p)}(\epsilon ; z, \cdot)\right)-\int_{\partial \mathcal{D} \backslash S} u \wedge\left(R_{q+1}^{(p)}\left(v_{1}\right)-R_{q+1}^{(p)}(\epsilon ; z, \cdot)\right) \\
& +\bar{\partial} h_{q}^{(p)} u(z)
\end{aligned}
$$

for $z \in U \cap \mathcal{D}$. This equality holds for all positive $\epsilon$ close to zero. Moreover, both the left-hand side and the last summand on the right-hand side are independent of $\epsilon$. As the restriction of $u$ to $\partial \mathcal{D} \backslash S$ is continuous and $R_{q+1}^{(p)}(\epsilon ; z, \cdot)$ approximates $R_{q+1}^{(p)}(z, \cdot)$ in the norm of $L^{1}(\partial \mathcal{D} \backslash S)$, we may assert that the integral over $\partial \mathcal{D} \backslash S$ on the right-hand side tends to zero when $\epsilon \rightarrow 0$. Thus, passing to the limit when $\epsilon \rightarrow 0$ we arrive at formula (3.1), as desired.

The difference $C_{q+1}^{(p)}(\epsilon ; z, \zeta)=K_{q+1}^{(p)}\left(v_{1}\right)-R_{q+1}^{(p)}(\epsilon ; z, \zeta)$ is said to be a Carleman function for the Dolbeault cohomology of $\mathcal{D}$ at step $q$. Let us observe that

$$
\begin{aligned}
\bar{\partial}_{\zeta} C_{q+1}^{(p)}(\epsilon ; z, \zeta) & =\bar{\partial}_{\zeta} K_{q+1}^{(p)}\left(v_{1}\right) \\
& =(-1)^{p+q} \bar{\partial}_{z} K_{q}^{(p)}\left(v_{1}\right)
\end{aligned}
$$

for all $(z, \zeta) \in(U \cap \mathcal{D}) \times \mathcal{D}$, the last equality being due to Lemma 1.2.

Corollary 3.2 Suppose there exists a Carleman function for the Dolbeault cohomology of $\mathcal{D}$ at step $q$. If $u \in C^{1}\left(\Lambda^{p, q} T_{\mathbb{C}}^{*} \overline{\mathcal{D}}\right)$ is $\bar{\partial}$-closed in $\mathcal{D}$ and $\bar{\partial}$-exact on $S$, then $u$ is $\bar{\partial}$-exact in $U \cap \mathcal{D}$.

Proof. Let $u=\bar{\partial} v$ on $S$ where $v \in C^{1}\left(\Lambda^{p, q-1} T_{\mathbb{C}}^{*} \bar{S}\right)$. Integrating by parts and using (3.2) we get

$$
\begin{aligned}
\int_{S} u \wedge C_{q+1}^{(p)}(\epsilon ; z, \cdot) & =\int_{S} \bar{\partial} v \wedge C_{q+1}^{(p)}(\epsilon ; z, \cdot) \\
& =\int_{\partial S} v \wedge C_{q+1}^{(p)}(\epsilon ; z, \cdot)+\bar{\partial} \int_{S} v \wedge K_{q}^{(p)}\left(v_{1}\right)
\end{aligned}
$$

for all $z \in U \cap \mathcal{D}$. Since the restriction of $v$ to $\partial S$ is continuous and $C_{q+1}^{(p)}(\epsilon ; z, \cdot)$ converges to zero in the $L^{1}(\partial S)$-norm when $\epsilon \rightarrow 0$, we conclude that the integral over $\partial S$ on the right-hand side tends to zero as $\epsilon \rightarrow 0$. It follows that

$$
u=\bar{\partial}\left(\int_{S} v \wedge K_{q}^{(p)}\left(v_{1}\right)+h_{q}^{(p)} u\right)
$$


in $U \cap \mathcal{D}$, proving the corollary.

The question of the existence of a Carleman function for the Dolbeault cohomology is at present far from being solved. We just mention that such is the case if $H^{n-q-1}\left(\mathcal{E}\left(\Lambda^{n-p, \cdot} T_{\mathbb{C}}^{*} \overline{\mathcal{D}}\right)\right)$ is dense in $H^{n-q-1}\left(\mathcal{E}\left(\Lambda^{n-p \cdot \cdot} T_{\mathbb{C}}^{*}(\partial \mathcal{D} \backslash S)\right)\right)$. This latter condition is actually equivalent to the dense solvability of the transpose to the Cauchy problem for the Dolbeault cohomology of $\mathcal{D}$ at step $q$ with data on $S$.

\section{Support function}

In this section we show how Lemma 1.3 can be used to derive explicit Carleman functions for the Dolbeault cohomology of $\mathcal{D}$. To this end the concept of a 'support function' for the Dolbeault cohomology of $\mathcal{D}$ proves useful. We keep the notation of the previous sections. In particular, $U$ is a neighbourhood of $\partial \mathcal{D} \backslash S$ in $\mathbb{C}^{n}$.

Definition 4.1 A smooth mapping $v:(U \cap \mathcal{D}) \times(\partial \mathcal{D} \backslash S) \rightarrow \mathbb{C}^{n}$ satisfying $\langle v, \zeta-z\rangle=1$ is said to be a support function for the Dolbeault cohomology of $\mathcal{D}$ at step $q$ if $\bar{\partial}_{\zeta} D_{1, q, n-q-1}\left(v, \bar{\partial}_{z} v, \bar{\partial}_{\zeta} v\right)=0$ for all $(z, \zeta) \in(U \cap \mathcal{D}) \times(\partial \mathcal{D} \backslash S)$.

Usually one considers support functions of the form $v\langle v, \zeta-z\rangle^{-1}$ where $v:(U \cap \mathcal{D}) \times(\partial \mathcal{D} \backslash S) \rightarrow \mathbb{C}^{n}$ satisfies $\langle v, \zeta-z\rangle \neq 0$ pointwise on the set $(U \cap \mathcal{D}) \times(\partial \mathcal{D} \backslash S)($ cf. Ch. $\mathrm{V}$ in Range $[\operatorname{Ran} 86])$.

Example 4.2 Assume that the domain $\mathcal{D}$ is linearly concave close to $\partial \mathcal{D} \backslash S$ in the sense that for each point $z \in U \cap \mathcal{D}$ there is a complex hyperplane $\left\{\zeta \in \mathbb{C}^{n}:\langle v(z), \zeta-z\rangle=0\right\}$ through $z$ which does not intersect the set $\partial \mathcal{D} \backslash S$. Then $v(z)\langle v(z), \zeta-z\rangle^{-1}$ is a support function for the Dolbeault cohomology of $\mathcal{D}$ at step $q$, for each $q$.

If $v_{0}$ is a support function for the Dolbeault cohomology of $\mathcal{D}$ at step $q$, then the double differential form $K_{q+1}^{(p)}\left(v_{0}\right)$ is $\bar{\partial}$-closed in $\zeta \in \partial \mathcal{D} \backslash S$, for any $z \in U \cap \mathcal{D}$. Hence equality (1.3) yields an explicit decomposition (2.1) with

$$
\begin{aligned}
R_{q+1}^{(p)}(z, \zeta) & =K_{q+1}^{(p)}\left(v_{0}\right)-(-1)^{p+q} \bar{\partial}_{\zeta} I_{q+2}^{(p)}\left(v_{t}\right), \\
P_{q+1}^{(p)}(z, \zeta) & =(-1)^{p+q} I_{q+1}^{(p)}\left(v_{t}\right),
\end{aligned}
$$

where $v_{t}=(1-t) v_{0}+t v_{1}$. Thus Theorem 3.1 still applies with $R_{q+1}^{(p)}(z, \zeta)$ and $P_{q+1}^{(p)}(z, \zeta)$ given by $(4.1)$.

Note that $R_{q+1}^{(p)}(z, \zeta)$ and $K_{q+1}^{(p)}\left(v_{0}\right)$ differ by a $\bar{\partial}_{\zeta}$-closed double differential form, and so the problem of approximating $R_{q+1}^{(p)}(z, \zeta)$ reduces easily to the analogous problem for $K_{q+1}^{(p)}\left(v_{0}\right)$. 


\section{References}

[AD75] L. A. Aizenberg and Sh. A. Dautov, Differential Forms Orthogonal to Holomorphic Functions or Forms, and Their Properties, Amer. Math. Soc., Providence, R.I., 1983.

[AG62] A. Andreotti and H. Granert, Théorèmes de finitude pour la cohomologie des espaces complexes, Bull. Soc. Math. France 90 (1962), $193-259$.

[AH72a] A. Andreotti and C. D. Hill, E.E.Levi convexity and the Hans Lewy problem. Part 1: Reduction to vanishing theorems, Ann. Scuola Norm. Super. Pisa 26 (1972), no. 3, 325-363.

[AH72b] A. Andreotti and C. D. Hill, E.E.Levi convexity and the Hans Lewy problem. Part 2: Vanishing theorems, Ann. Scuola Norm. Super. Pisa 26 (1972), no. 4, 747-806.

[Aiz93] L. A. Aizenberg, Carleman Formulas in Comlex Analysis. First Applications, Kluwer Academic Publishers, Dordrecht NL, 1993, $358 \mathrm{pp}$.

[AT90] L. A. Aizenberg and N. N. Tarkhanov, Conditionally stable problems and Carleman formulas, Sibirsk. Mat. Zh. 31 (1990), no. 6, $9-15$.

[Ker72] N. Kerzman, The Bergman kernel function. Differentiability at the boundary, Math. Ann. 195 (1972), no. 2, 149-158.

[Kop67] W. Koppelman, The Cauchy integral for differential forms, Bull. Amer. Math. Soc. 73 (1967), no. 4, 554-556.

[Lau75] H. Laufer, On the infinite dimensionality of the Dolbeault cohomology groups, Proc. AMS 52 (1975), 293-296.

[Ran86] R. M. Range, Holomorphic Functions and Integral Representations in Several Complex Variables, Graduate Texts in Mathematics (No 108), Springer-Verlag, Berlin et al., 1986.

[Tar95] N. N. Tarkhanov, Complexes of Differential Operators, Kluwer Academic Publishers, Dordrecht, NL, 1995. 